\title{
Revisión del problema ambiental y su gestión
}

\author{
Fecha de recepción: 20/10/2009 Aceptación: 05/04/2010
}

\section{Clemencia Camacho Delgado y Pedro Pablo Cardoso}

\section{Resumen}

En este artículo se hace una revisión histórica desde el siglo III antes de Cristo con Eratóstenes, quien podría considerarse el que dejó el primer registro del fenómeno de la erosión en la isla de Chipre. El recorrido muestra concepciones y hechos de la cultura occidental en el siglo XIII hasta el periodo industrial moderno del siglo XIX, en el que se presenta un aumento de textos científicos relacionados con los efectos negativos en la salud o en el medio ambiente. Posteriormente se hace mención a los sistemas de gestión ambiental, más adelante presentados en la norma ISO 14000. Finalmente se presenta la problemática ambiental de los centros de desarrollo académico y se incluye un cuadro secuencial de las iniciativas y eventos realizados por universidades desde los años 1950. Se busca llegar a entender el tema ambiental como un elemento orientado hacia la solución de problemas con relevancia a lo local en una mirada global.

\begin{abstract}
This paper offers a review of the environmental problem and its management since the third century $A C$ to the latest development in environmental management in the XXI century. The review presents the conceptions and facts that shaped the relationship among the western industrial civilization and the environment. This paper ends with comments about the environmental management systems and the participation of the academic sector. This paper is aimed to present the environmental management as a values-oriented issue drived towards the solution of local problems within a global perspective.
\end{abstract}

\section{Palabras clave}

Contaminación, medio ambiente, gestion ambiental, interdisciplinaridad.

\section{Keywords}

Environmental pollution, environment, environmental management, interdisciplinary.

\section{Tempranas evidencias de la problemática ambiental y su registro}

Los diferentes problemas ambientales que se han detectado en la historia reciente de la humanidad, como el calentamiento global y la disminución de la capa de ozono, han tenido por respuesta manifestaciones de interés en diferentes instancias en el ámbito internacional, cuya evolución se evidencia en el surgimiento de algunos organismos de control multilateral, la expresión de 
políticas internacionales, y regulaciones de la actividad industrial asociada al desempeño ambiental mediante la generación de marcos normativos e institucionales para la solución de este problema. Como prueba de ello se presenta a continuación un recorrido histórico que pretende ilustrar la forma como surgieron los diferentes sistemas de gestión ambiental.

Si nos remontamos a los inicios de la historia de la cultura occidental, en el siglo III a. C. -según la descripción hecha por Hutchinson y Hutchinson (1997)Eratóstenes encargado de la Biblioteca de Alejandría, realizó el que podría considerarse el primer registro del fenómeno de la erosión en la isla de Chipre como consecuencia de la tala insostenible allí realizada para la construcción de navíos, fundición

\section{Reseñas de autores \\ Clemencia Camacho Delgado}

(Politécnico Grancolombiano)

ccamacho@poligran.edu.co

Bióloga y MSc. de la Pontificia Universidad Javeriana, con especialización en Gestión Ambiental de la Universidad Externado de Colombia. Diplomada en Docencia Universitaria, Enfoques Pedagógicos Contemporáneos, Investigación, y Estudios de China Contemporánea. Cuenta con varios artículos publicados en revistas universitarias. Directora de Investigación del Politécnicos Grancolombiano y del grupo Educación e Investigación de la institución.

\section{Pedro Pablo Cardoso}

ppccardoso2@gmail.com

Biólogo Marino de la Universidad Jorge Tadeo Lozano con Máster en Planeación y Auditoría Ambiental Empresarial del Instituto de Investigaciones Ecológicas de Málaga (España) y Máster en Comercio Internacional del Instituto de Estudios Bursátiles de la Universidad Complutense - Bolsa de Madrid. Durante los últimos diez años ha combinado su actividad académica como catedrático-investigador en diferentes programas de pre y posgrado con sus actividades de emprendimiento y consultoría en sistemas integrados de gestión (ISO 9000-14000), negocios internacionales y mercadotecnia. Cursa su tercer año de estudios de doctorado en la Business School de la Universidad de Hull (Inglaterra), de cobre y extracción de plata. Diagnosticó también los efectos de la erosión, que se constataban en la sedimentación de los ríos y la disminución de la pesca; hecho que fue también documentado por geógrafos y filósofos griegos como Platón y Aristóteles.

Con el transcurrir del tiempo, las civilizaciones se desarrollaron, lo cual implicó el uso cada vez mayor de recursos naturales y la aparición de problemas ambientales a los que las mismas sociedades que los generaban presentaron, cada una a su manera, diferentes soluciones y respuestas. Como ejemplo de lo anterior se pueden registrar algunos eventos desde el siglo III a. C., hasta la aparición de las primeras figuras normativas en el siglo XIII. Entre ellos están las memorias de los cronistas romanos del 312 a. C. donde se habla de la contaminación del río Tiber y cómo las colonias romanas asentadas en sus márgenes tuvieron que buscar nuevas fuentes de agua potable.

En el siglo XIII la mayoría de las grandes ciudades de Europa tenía algún grado de contaminación grave, como el caso de Siena (Italia), nacida en la cima de tres colinas y circundada por los bien conservados muros de cinta, en cuya Piazza del Campo se utilizaban cerdos como consumidores de los desechos de esta. La situación llegó a ser tan grave que en 1366 las autoridades desalojaron a los carniceros de la ciudad debido a que "el Siena no es capaz de asimilar la gran cantidad de despojos que recibe". Circunstancias similares fueron frecuentes en las ciudades europeas hasta el periodo comprendido entre 1451 al 1648 , momento en que se presentaron las primeras leyes específicas, particularmente en Inglaterra, cuya aplicación no fue efectiva por parte de los organismos encargados de su ejecución (Wolman, 1956). 
Al final del siglo XVI, los filósofos, naturalistas y científicos europeos redescubrieron conocimientos acumulados por diversas culturas y civilizaciones con relación al medio ambiente; entre ellos se destacó el naturalista Georges-Louis Leclerc, conocido como Comte Buffón (1707-1788), quien describió en numerosos tratados los efectos causados en la naturaleza por las prácticas de domesticación y selección artificial de plantas y animales (Mayr, 1981).

Con el surgimiento del concepto nación -Estado, en el siglo XVII las nuevos formas sociales y los ordenamientos territoriales suscitaron la creación de estamentos burocráticos que entre otras actividades, trataban de regular las relaciones entre el hombre y el medio ambiente como, por ejemplo, la ordenanza forestal de Colbert, en Francia.

En ese mismo momento histórico, los científicos establecieron e identificaron relaciones de causalidad entre algunas enfermedades y el desarrollo industrial. Ejemplo de ello es la relación existente entre la enfermedad del ceramista y el envenenamiento por plomo; la relación entre los cuadros clínicos de los sombrereros y el envenenamiento por mercurio y las lesiones pulmonares y bucales de los artesanos del vidrio con la utilización de bórax y antimonio, diagnosticadas por el médico Bernardino Ramazzini, quien fue profesor de medicina práctica en las universidades de Modena y Padua entre 1682 y 1714 (Bisetti, 1988; Hook, 1995).

Con la llegada del siglo XIX y el periodo industrial moderno, aumentaron textos científicos que relacionaban los efectos negativos en la salud y el medio ambiente con la Revolución Industrial, siendo destacable por sus futuras implicaciones (a finales del siglo XX), el de la contaminación del aire y la lluvia ácida, observado en Inglaterra en 1859 por Robert Smith.

Debido a estos documentos científicos y a la preocupación pública por asuntos relacionados con la actividad industrial y la calidad ambiental, algunos gobiernos promulgaron normas para ejercer control directo sobre las empresas, como fue el caso en 1821 cuando el Parlamento británico, pretendiendo facilitar el proceso de denuncia y demanda ante las autoridades contra los dueños de los hornos de fundición que emitían cantidades excesivas de partículas, emitió una norma regulatoria; o el caso en 1863 en el que el Consejo Británico promulgó la norma-reglamento para la prevención de la contaminación conocida como el "Decreto Alcalino", que exigía a los fabricantes de productos alcalinos -empleados en la fabricación de jabón, vidrio y textiles- la eliminación del 95\% del ácido clorhídrico emitido por sus fábricas. Así estableció la primera entidad de control y verificación de la contaminación registrada en la historia hasta ese momento, conocida como la Álcali-Inspectorate (Thomas, 1956).

La migración proveniente de zonas rurales que acompañó el crecimiento de la revolución industrial fue un fenómeno común a la gran mayoría de centros urbanos en donde se concentró la actividad industrial, que para la década de 1850 motivó las primeras medidas de gestión de residuos urbanos evidenciados en los trazados de los grandes proyectos de alcantarillado de Hamburgo (1842), Chicago (1855) y Berlín (1860). Por ejemplo, sobre el desarrollo de sistemas de alcantarillado en grandes centros urbanos, tal vez el más impresionante en virtud a su antigüedad, reto tecnológico y cantidad de población atendida fue el de la ciudad de Roma que 
llegó a su apogeo a finales de la Era Dorada (República) (Clarence, 1956).

\section{Impactos industriales en el medio ambiente}

En esta misma década, aparecen obras que bien podrían considerarse pioneras en el establecimiento de los fundamentos de la ecología, al tratar desde un punto de vista holístico sobre las interrelaciones del hombre y la naturaleza, de las que se destacan las de Ernst Heinrich Heackel que en 1866 empleó por primera vez el término “ecología”, y que en su obra de 1848 titulada Physical Geography describía las leyes de la ecología.

Por estas mismas fechas, en Estados Unidos, el desarrollo industrial llevó a la desaparición de las áreas vírgenes; razón por la cual en 1864 se creó el decreto de Conservación de Yosemite, que permitió la conservación de la zona central del estado de California que se convirtió en la primera reserva natural (Parque Natural) del mundo (Muir, 1962).

Mas tarde, Ernst Friedrich, geógrafo alemán, describió la necesidad de apoyar el desarrollo económico sostenible en 1904, haciendo la distinción entre economías de extracción simple entendidas estas por aquellas que no causan daños permanentes; y las economías de extracción total, que destrozan el medio ambiente hasta el punto de producir el empobrecimiento de un pueblo (tema que sería retomado setenta años después) (Naredo, 1993)

El establecimiento creciente de áreas protegidas se acompañó de estudios económicos, de modo que entre 1900 y 1950, se publicaron estudios de este tipo relacionados con la valoración de la contaminación. El economista K. William Kapp por ejemplo, cita varios trabajos realizados en Estados Unidos y Gran Bretaña entre 1914 y 1930 dentro de los cuáles se encuentran intentos por definir los costes de la contaminación del aire, como una primera aproximación a la determinación del concepto de "externalidad" (Kapp, 1950; Kapp, 1950a).

En Inglaterra se desarrollaron trabajos similares desde las ciencias sociales, de modo que en 1942, Massingham H.J. publicó The English Countryman: a Study of the English Tradition, que analiza las implicaciones de la desvinculación de la gente con la tierra, y describe en detalle la presión a la que eran sometidos los suelos al pasar de prácticas tradicionales de agricultura balanceada a prácticas de monocultivo intensivas. El uso ilimitado de maquinaria así como la motivación egoísta de lucro como estímulo a la labor en el campo conducían a la pérdida y al desperdicio de capital en la forma de fosfatos y demás abonos requeridos para mantener su capacidad de producción generando de este modo un deterioro económico acumulado.

Estos hechos hicieron que en $1952 \mathrm{el}$ geógrafo Carl Sauer, el sociólogo Lewis Mumford (1961) y el zoólogo Marson Bates, reclamaran a la comunidad académica internacional el reconocimiento y el estudio sistemático de las consecuencias que sobre el planeta y la biosfera generaba el desarrollo de las diversas culturas y civilizaciones existentes. Su iniciativa y liderazgo permitió que en 1955 se celebrara del 16 al 20 de junio de 1955 en Princeton, New Jersey, la conferencia titulada "El papel del hombre en los cambios de la faz de la tierra", reunión que contó con la participación de de meteorólogos, antropólogos y otros científicos sociales de renombre mundial, 
en la que se discutió el papel del desarrollo industrial en el deterioro ambiental.

\section{Década de los sesenta: se afianza preocupación cientifica}

Esta década presentó algunas de las más impactantes y determinantes manifestaciones científicas y de opinión pública con relación a la problemática ambiental. Entre ellas, catalogable como precursora de muchas otras iniciativas y acciones pro ambientalistas, se encuentra la publicación de Rachel Carson La primavera silenciosa (1962), un texto científico por medio de una narrativa elocuente despertó la preocupación de buena parte de la población de Estados Unidos con relación a los efectos nocivos sobre la fauna y los seres humanos, de sustancias como el diclorodifenil-tricloroetano (DDT), y logró que personas ajenas a la formación y discusión científica entendieran el concepto holístico de la ecología. El impacto de su estudio fue tan grande que fue traducido a más de una docena de idiomas y generó una conciencia global alrededor de la problemática ambiental y de los daños asociados a la producción y uso de sustancias tóxicas (Dasman, 1965).

Esta obra generó reacciones polémicas ampliamente documentadas, entre ellas las escandalosas contrataciones de científicos por parte de industriales para desmentir el trabajo de la doctora Carson, y de publicaciones con comentarios en los que se afirmaba que ella no estaba capacitada para hacer tales observaciones calificándola de histérica y loca, o afirmando incluso que las mujeres están impedidas para entender las relaciones complejas de los sistemas naturales (Ponting, 1991).

Esta publicación coincidió con el lanzamiento de la Ecología, ciencia interdiscipli- naria y moderna que motivó por primera vez la creación de programas educativos en todos los niveles con contenidos ambientales de rigor académico. Hecho que fue acompañado por muchas otras publicaciones como The Destrucción of California, America the Raped y The Greening of America, que con rigor científico, pero en un lenguaje asequible al común de la gente, exponían a lectores no formados en el campo de las ciencias naturales, una serie extensa de problemas ambientales, su relación con la actividad industrial, y sus dramáticas repercusiones sobre la salud humana (Marine, 1969; Reich, 1970).

El resultado fue el aumento de la preocupación pública y tras ella una fuerte presión ejercida de manera creciente por una población que cada vez más tenía fácil acceso a la información relacionada con problemas ambientales y a sus efectos sobre la salud, de modo que en respuesta a esta situación, el estado de California estableció la Agencia para la Protección del Medio Ambiente (EPA, Environmental Protection Agency).

Esta agencia para 1969 creció en importancia hasta convertirse en una institución federal mediante la política nacional del medio ambiente (The Nacional Environmental Policy Act), cuya función consistía en monitorear el impacto ambiental de todas las obras o instalaciones de gran tamaño, y establecer parámetros de control y auditoría, las cuales se adelantaron por cerca de veinte años según los protocolos definidos por las normas británicas BS7750, e ISO 14000, como se verá más adelante .

Un año antes, ocurría en el contexto internacional un evento que definiría el futuro de la orientación ideológica de la gestión ambiental, en 1968 se invitó a las
Catalogable como precursora de muchas otras iniciativasy acciones pro ambientalistas, se encuentra la publicación de Rachel Carson La primavera silenciosa (1962) 
En 1973 la

Comunidad

Europea

estableció

el primer

programa

de acción

ambiental

aplicado hasta

1976 y que se

constituyó en

uno de los más

importantes

e influyentes

sistemas de

regulación

gubernamental. potencias mundiales a discutir sobre la problemática ambiental (reunión del Club de Roma), donde se evidenció que no se disponía de información clara o suficiente para la formulación de planes estratégicos sobre esta problemática, y se delegó a la primer ministro noruega, la doctora Gro Brundtland, la conformación de un equipo interdisciplinario que diera respuesta a esta carencia de información (Griffiths, 2005)

\section{El medio ambiente: se vuelve objeto de estudio}

Los inicios de la década de los setenta marcaron una nueva dimensión en la preocupación por asuntos ambientales que para entonces se convertían en objeto de estudio no solo de entes académicos, sino industriales, y gubernamentales, (Dobson, Andrew, 1999). Prueba de ello fue la creación en Estados Unidos de la EPA (Environmental Protection Agency), a partir de la unión de quince agencias independientes que existían desde 1969. Este mismo año se publicó el libro de Barry Commoner (1971) titulado The Closing Circle, Nature, Man, and Technology, que expone los principios de la ecología moderna; así se institucionalizaba la Ecología como una ciencia aceptada en el contexto global.

Otro caso destacable en el contexto empresarial es el de IBM, que en 1971 estableció su política ambiental corporativa, siendo una de las primeras empresas en el mundo en hacerlo. Dentro de las iniciativas oficiales, en 1972 tuvo lugar en Estocolmo, la primera Conferencia del Medio Ambiente Humano, Desarrollo Económico y Medio Ambiente, patrocinada por la ONU donde se estableció la creación del Programa de las Naciones Unidas para el Medio Ambiente (UNEP-PNUMA).
En este contexto se trató por primera vez, en el ámbito diplomático internacional, la problemática de la contaminación asociada al desarrollo industrial y se emprendieron estudios sistemáticos sobre las consecuencias globales del progreso y el desarrollo industrial.

Ese mismo año, como resultado de esta conferencia mundial, se publicó el informe titulado Los limites del crecimiento, en el que se estipulaba la urgencia de detener el ritmo del crecimiento económico, entendido como consumo intensivo de bienes primarios de capital para la generación de riqueza. Así mismo, se demandaba de la comunidad industrial internacional un cambio severo en los paradigmas existentes de bienestar, crecimiento económico y desarrollo, pues predecía que, de no efectuase tales cambios en el sistema de producción global, se llegaría a una situación de escasez global de recursos que conduciría al colapso de los intercambios comerciales y el derrumbamiento del sistema industrial (Bruntland, 1987).

En 1973 la Comunidad Europea estableció el primer programa de acción ambiental aplicado hasta 1976 y que se constituyó en uno de los más importantes e influyentes sistemas de regulación gubernamental. Se realizaron programas posteriores de intervención estatal sobre asuntos ambientales cada cuatro años conservando y mejorando la estructura básica de referencia establecida en este primer acto de política pública ambiental de la Comunidad Europea.

Uno de los aportes de esta iniciativa fue la publicación de directrices para el uso de pesticidas, etiquetado y mercadotecnia. Regulaciones que tuvieron mayor impacto en las legislaciones nacionales de los países miembros; donde las observaciones sobre 
etiquetado de productos y normas para el mercadeo de bienes con atributos favorables para el medio ambiente, llamaron la atención a finales de la década como instrumento de diferenciación de producto y tendencia emergente en las estrategias de mercadeo.

Como resultado de esta nueva tendencia en 1978 apareció en Alemania el esquema de etiquetado conocido como "ángel azul", basado en la declaratoria de las Naciones Unidas, y con el aval del Instituto Alemán para el Aseguramiento de la Calidad y la Oficina Federal de Medio Ambiente. Con el que se pretendía motivar a los fabricantes a suministrar al mercado productos ambientalmente amigables.

Este programa de etiquetado no realizaba una verificación de conformidad y no pasó mucho tiempo antes de que los europeos descubrieran que la comercialización de los productos "inocuos" para el medio ambiente no tenía mucho que ver con las políticas de medio ambiente de los países miembros.

En 1979 como resultado de estas y muchas otras iniciativas de intervención gubernamental, el British Standard Institute (BSI), publicó la serie de normas de calidad BS 5750. Estas normas presentaban por primera vez, lo que se entiende por un sistema de gestión corporativo, tomando como modelo para su creación los conceptos de mejora continua que desde los años 1950 habían sido expuestos y validados en la práctica de la industria japonesa por Edward W. Deming. Estas disposiciones darían pie luego a la edición de un nuevo paquete de normas ambientales más parecidas a lo que entendemos hoy como sistemas de gestión ambiental certificables.

\section{De 1980 a 2000: indicios de daños industriales al medio ambiente}

Los impactos del crecimiento de la población y de los volúmenes de la producción industrial fueron evaluados en 1980 por la Unión Internacional para la Conservación de la Naturaleza (UICN), United Nations Environment Programme (UNEP) y World Wild Fundation (WWF), 1991. Este informe indicaba que desde la Revolución Industrial, el tamaño de la población mundial se ha multiplicado por ocho, que la producción industrial se ha multiplicado por cien en el paso de cien años, y que los efectos ambientales tienen mucho que ver con estos cambios globales. Además indicaba que si la productividad y la vitalidad del planeta no son salvaguardadas, el futuro de la humanidad estará amenazado. Asimismo establecía el concepto de "desarrollo sostenible" como única opción racional para garantizar nuestra supervivencia, observación que de inmediato conmocionó a la comunidad internacional.

Posteriormente entró en escena de nuevo la ONU con la publicación del Informe Mundial 2000, bajo el mandato en Estados Unidos del Presidente Jimmy Carter; este informe no fue acogido en Estados Unidos debido a la derrota electoral tendiente a la reelección, pero su repercusión internacional fue innegable al distribuirse más de millón y medio de copias con traducción a más de ocho idiomas. Este documento es una aproximación a la primera política internacional de medio ambiente, que trataba sobre las existencias o stocks de bienes ambientales estratégicos para el ulterior desarrollo económico e industrial de las economías de Occidente. Explora la idea de las reservas naturales de la biodiversidad como fuente de 
la futura riqueza, las fuentes de agua y, por supuesto, las existencias de recursos energéticos como prioridades para la futura administración del desarrollo.

En 1982 en Seveso (Italia), ocurrió una tragedia ambiental ocasionada por un derrame de productos químicos, que generó una enérgica reacción por parte de las agencias encargadas del control ambiental de la Comunidad Europea. De este modo, estos órganos gubernamentales emitieron el mismo año, la primera directiva comunitaria (82/501/CEE, conocida como Seveso) para el control de actividades peligrosas.

En 1984 ocurrió otro accidente de gran magnitud. En esa ocasión un incendio en las bodegas de un almacén de la empresa Sandoz en Bale (Suiza) ocasionó un derrame de sustancias tóxicas al río Rin, con daños desde Suiza hasta Holanda, que afectó a la industria pesquera de las naciones vinculadas al río y a la desembocadura del mismo en el mar, los cuales mostraron a los líderes políticos que la contaminación no conoce fronteras y demostraron en forma dramática la importancia de los sistemas de contención.

Estos hechos motivaron múltiples directivas de la Comunidad Europea tendientes a regular e imponer mecanismos para la protección ambiental que debían ser adoptadas por los gobiernos locales. Por esta razón Francia adoptó en 1984 la directiva Seveso; Dinamarca y Alemania establecieron normas para el agua, las cervezas y los refrescos según la cual, estos solo podían distribuirse en empaques reutilizables, lo cual generó protestas ante el tribunal de la Unión Europea porque las calificaba como medidas contra el libre comercio. Años más tarde (1992) el fallo sancionó la tendencia actual en términos de regulación comercial y protección ambiental al afirmar que "el principio de protección al medio ambiente está por encima del principio de libre comercio" que garantiza la Unión Europea.

\section{Medidas de protección al medio ambiente}

Ante la presión ejercida por la sociedad, diferentes entidades lideraron la propuesta de establecer estructuras lógicas que sistematizaran la gestión ambiental en las industrias, haciéndolas compatibles con los intereses de los clientes por la calidad; y de la gerencia, por la productividad y la eficiencia. Fue así como se presentaron diferentes programas por parte de la Cámara de Comercio Internacional, de los gremios industriales y de algunos organismos de estandarización internacionales (Vogel, 1990).

Uno de los primeros resultados de esta concientización fue el desarrollo del programa Responsible Care por parte de la Asociación de Industrias Químicas en 1984. Los resultados más concretos relacionados con esta iniciativa se obtuvieron con la publicación de la Carta Empresarial para el Desarrollo Sostenible, elaborada por la Cámara de Comercio Internacional, en 1990 y con la creación de la Norma Británica BS7750 "Specification for Environmental Management Systems" por parte de la British Standard Institution.

En 1985 se realizó la cumbre económica de Estocolmo, que analizó el deterioro de la economía de la península escandinava por causa de la lluvia ácida, - generada por las chimeneas de la industria pesada inglesa-, a la cual la primer ministra británica no asistió. A pesar de ello, se tomaron medidas inmediatas como regular la quema de 
carbón en la industria pesada y cerrar las acerías del norte de Inglaterra.

Como resultado de la difusión de informes sobre el estado del medio ambiente al inicio de la década del ochenta (Informe Mundial, entre otros.), y dados los avances tecnológicos en la identificación de problemáticas ambientales de carácter transnacional, se celebró en 1987 el Protocolo del Ozono de Montreal, reunión económica citada con carácter urgente por el presidente de Estados Unidos, Ronald Reagan y la primer ministro inglesa Margareth Tatcher. Además fueron convocados a ella todos los mandatarios de las economías occidentales y asiáticas para exponer la situación de la capa de ozono, identificada en las primeras imágenes satelitales de los casquetes polares que diagnosticaban el deterioro de esta capa protectora de la atmósfera y cuyos efectos repercutieron en la salud humana y se hicieron patentes por la presencia de casos epidémicos de cáncer de piel en colonias británicas del Pacífico Sur y en Australia.

Esta cumbre económica tuvo el propósito de establecer un mecanismo efectivo e inmediato que regulara la emisión de sustancias que afectaran la capa de ozono. Consiguió, asimismo, establecer objetivos cuantitativos específicos, la sustitución gradual y eliminación de sustancias como clorofluorocarburos (CFC) y fluorocarburos (FC), cuyos resultados obtenidos a corto plazo marcaron un hito al hacer de esta una de las iniciativas de la comunidad internacional más efectivas en términos de sustitución y renovación del aparato productivo en pro de la protección del medio ambiente global (McCormick, 2002)

Este mismo año, aparece de nuevo en escena la ONU a través de la Comisión
Mundial de Medioambiente y Desarrollo, liderada por la doctora Gro Harlem Bruntland, con la publicación del que ha sido, tal vez, el documento con más impacto en la definición de políticas económicas, regulatorias y estatales relacionadas con el manejo de la naturaleza y de los recursos naturales. Este fue el Informe de la Comisión Bruntland titulado: "Nuestro futuro común" (conocido también como Informe Bruntland).

Este documento establece una gran agenda para el cambio, sugiriendo con vehemencia la adopción e implementación de estrategias ambientales a partir de un enfoque holístico de largo plazo, la exploración de las complejas relaciones entre los pueblos, de los recursos, del medio ambiente y del desarrollo económico. Estos aspectos son considerados como esenciales para el desarrollo e implementación de estrategias de cooperación y beneficio mutuo de la comunidad internacional; y reitera el concepto de "desarrollo sostenible" (sociedad sostenible), definido en este documento como:

La sociedad que satisface las necesidades de las presentes generaciones sin comprometer la posibilidad de que las generaciones futuras satisfagan las suyas propias.

En respuesta a este informe, algunos países de la actual Comunidad Europea, iniciaron el rediseño de sus aparatos productivos y la normatización de su actividad industrial incluyendo el componente ambiental en estas incipientes planeaciones.

\section{Adopción de propuestas, políticas y planes}

Entre las actividades de este especial año, es importante mencionar la unión entre John Elkington, quien desde 1974 había estu-
La sociedad que satisface las necesidades de las presentes generaciones $\sin$ comprometer la posibilidad de que las generaciones futuras satisfagan las suyas propias. 
diado la relación entre medio ambiente, desarrollo y negocios, y Julia Hailes, para trabajar en los proyectos de Earthlife Fundation. Ellos propusieron el término "consumidor verde" que causó gran aceptación en el consejo de diseño del Reino Unido.

Luego de los eventos anteriores, y motivado por estos, en 1987 el gobierno holandés, inspirado por las recomendaciones del Informe Bruntland, implementó el plan nacional de política ambiental (NEPP), conocido como el Plan Verde.

Con este plan se pretendía unificar de manera coordinada los esfuerzos de todos los ministerios y agencias para lograr la calidad del medio ambiente desarrollando metodologías de gestión centradas en el ecosistema más que en el aparato productivo, y con una concepción sensata de los recursos, la población y la actividad industrial, delimitó ocho áreas de intervención primordiales: (1) el cambio climático, (2) la acidificación, (3) la eutrofización, (4) las sustancias contaminantes tóxicas o peligrosas, (5) la disposición de residuos, (6) los efectos negativos en la calidad ambiental y de vida, (7) el agotamiento de los recursos hídricos, y (8) la pérdida de otros recursos.

Para ello se fijaron objetivos y metas nacionales de reducción en nueve sectores productivos delimitados: agricultura, industria, refinerías, comercio al por menor, transporte, consumidores, industria de la construcción, industria de tratamiento de aguas negras y los institutos de investigación.

Esta iniciativa holandesa se adelantó en su estructura a los requerimientos, que para sistemas de gestión ambiental más adelante serían presentados en la norma ISO 14000 en cuanto a la definición de objetivos, metas y programas de ejecución de estrategias corporativas tendientes a gestionar la capacidad de carga de los ecosistemas en los que se desenvuelve la actividad productiva de las empresas. El modelo del Plan Verde fue adoptado por Canadá, Nueva Zelanda y Singapur. Holanda, así como Canadá, abandonaron sus planes verdes a inicios de la década de los noventa.

Este año surgió la firma SustainAbility y John Elkington y sus colaboradores publicaron el libro The Green Capitalists en el cual, dos años antes de la caída del muro de Berlín, concluían que el futuro de la gestión ambiental estaba en los negocios y la dinámica del mercado (Elkington y Burke, 1987).

Al final de este decenio, en 1988 durante el discurso ante la Royal Society, el gobierno británico en cabeza de su primera ministra expuso el que luego sería conocido como Reporte Pearce titulado Blueprint for a Green Economy (Pearce, Markandya y Barbier, 1989). En este documento se presentó un resumen sobre el estado de las investigaciones relacionadas con el crecimiento económico y la preservación ambiental, así como su aparente incompatibilidad; y se recomendaba la combinación de instrumentos regulatorios y fuerzas de mercado para su gestión integral. La consecuencia de esta intervención fue la valoración de consideraciones ambientales en las políticas estatales a largo plazo, contemplando relaciones intra e intergeneracionales, y recomendaciones para generar instrumentos.

Este mismo año SustainAbility publicó el libro The Green Consumer, el cual posicionó a esta empresa dentro del listado 
mundial de agencias dedicadas a la promoción y creación de instrumentos para la gestión ambiental empresarial. En 1999 los fundadores de esta organización fueron nominados como una de las quinientas organizaciones ambientales más influyentes por sus "destacados logros ambientales"; ese mismo año, alcanzó a reclutar a sus primeros clientes internacionales (DowEuropa, Procter \& Gamble y Novo Nordisk) e inició sus labores con estas empresas. Con el reporte de Auditoría Ambiental con la WWF hizo de SustainAbility una de las empresas claves en la futura definición de los protocolos para auditorías de este tipo (Makover, Elkington y Hailes, 1993).

En 1989 el doctor Kart-Henrik Robert fundó en Suecia The Natural Step, un marco de trabajo fundamentado por información (mediciones) científica y teoría de sistemas que integra la dinámica social, económica y ambiental; así como las tendencias de la sociedad actual, en particular, en el uso de recursos naturales y emisión de toxinas. Este marco se realiza mediante una aproximación a la planeación corporativa para el logro de la sostenibilidad aportando para ello elementos de diseño de carácter analítico que pueden ser empleados para el direccionamiento de aspectos ambientales, sociales y económicos (Tippett, 2005).

Este mismo año y como consecuencia del desastre ambiental generado por el accidente del Exxon Valdes, motivó tanto a ambientalistas como a grupos de inversión a presionar por la aplicación de estándares más elevados de responsabilidad y desempeño ambiental corporativo. De esta situación coyuntural surge la Coalición para el Medio Ambiente y la Economía Responsable (Ceres, por su sigla en inglés), como la unión de grupos ambientalistas y grupos de inversionistas; y su declaración de principios Ceres, constituida por un decálogo de principios de conducta ambiental corporativa.

\section{La Cumbre de la Tierra: hito en la historia ambiental}

La década del noventa empezó con la planeación de la reunión cumbre de más trascendencia en el futuro desarrollo de la gestión ambiental internacional, que sería realizada en Río de Janeiro en 1992. Un año antes en Noruega, representantes de varios países se reunieron para establecer la forma como plantearían su posición en dicha cumbre. En este encuentro participó Stephan Schmidheiny, un industrial suizo con interés en vincular al sector privado en la discusión sobre temas relacionados con el desarrollo sostenible, y que como se verá adelante, tuvo gran influencia en la creación de uno de los órganos más comprometidos en la ejecución de los planes diseñados y expuestos en la cumbre de Río.

Uno de los resultados de esta reunión fue la invitación hecha a Stephan Schmidheiny por Maurice Strong, secretario general de la cumbre de Río, quien estableció como prioritario que este industrial suizo fuera coordinador del sector empresarial en la cumbre. Para esto, el señor Schmidheiny convocó a cerca de cincuenta gerentes de grandes empresas y conformó el que sería el comité de base del Consejo de Negocios para el Desarrollo Sostenible, cuya participación en la cumbre ambiental tendría dos propósitos: de un lado, llevar la opinión del sector productivo a las mesas de trabajo, y de otro, recopilar en un documento las opiniones de los empresarios durante la cumbre. 
En 1992 apareció la propuesta de gestión ambiental del Instituto Británico de Normas (British Standard Institute), que publicó la norma de adopción voluntaria BS 7750 con el título "Especificaciones para los sistemas de gestión medioambiental”. Esta respondía a la demanda del sector productivo europeo para normalizar la actividad industrial frente a los cada vez más exigentes requerimientos ambientales en desarrollo por el Reino Unido y la Comunidad Europea en general (Lamprecht, 1997).

Este mismo año se celebra la Conferencia Mundial de Medio Ambiente y Desarrollo de la ONU, conocida como la Cumbre de Río. De ella se emana un documento de trabajo y el plan de acción "Agenda 21", que reclama e institucionaliza el concepto de "desarrollo sostenible" (cuya definición no difiere sustancialmente de la sugerida en el Informe Bruntland). Allí se establecen compromisos tangibles para la comunidad internacional con relación a la gestión ambiental pública, y la participación directa del sector privado en la planeación de estas políticas, así como la asunción de responsabilidades en pro de la preservación del medio ambiente. Uno de los resultados más destacables de esta cumbre ambiental internacional fue el establecimiento de formas de producción más limpia como queda estipulado en el principio 8 de la declaración de principios fundamentales en la Agenda 21 (Muñoz, 2004).

Terminado el evento, el Consejo de Negocios y Medio Ambiente publicó las memorias de sus impresiones en el libro Changing Course: A Global Business Perspective on Development and the Environment. En él, se hace una introducción práctica a las nuevas formas de dirigir negocios atendiendo tanto a las dinámicas propias de los mercados como a las condiciones necesarias para preservar la calidad ambiental y los requerimientos del desarrollo humano. Basándose en la experiencia de los líderes de corporaciones multinacionales, junto con el soporte de numerosos estudios de casos, presenta las mejores prácticas empresariales demostrando, mediante un profundo análisis, cómo la comunidad de negocios puede adaptar y contribuir al logro del desarrollo sostenible al combinar los objetivos de protección ambiental y desarrollo económico, con la convicción de que solo permitiendo la libre operación de las fuerzas del mercado e integrando el principio de "quien contamina paga", las políticas económica y ambiental nos conducirán al logro del desarrollo sostenible.

En forma simultánea, la Comunidad Europea de Naciones unificaba sus criterios normativos y publicaba bajo la Resolución 1836 de 1993 la norma que reglamentaba la participación voluntaria de las compañías de los sectores industriales en el programa europeo de Eco Gestión y Eco Auditoría (Environmental Management and Audit Écheme, EMAS), en la cual algunos sectores productivos relacionados con altos niveles de generación de contaminantes debían implementar este sistema de gestión ambiental de manera obligatoria.

En 1993, aparece nuevamente SustainAbility, que en un proyecto conjunto con el Instituto Internacional para el Desarrollo Sostenible (IISD) publicó el libro Coming Clean, uno de los primeros referentes de los reportes ambientales corporativos. Se publica también el libro LCA Sourcebook, que une los conceptos de 
análisis de ciclo de vida con el diseño y el desarrollo de productos.

El año siguiente, esta misma firma de consultoría, liderando las que luego serían conocidas como las olas de cambio social con relación al consumo verde y a temas ambientales, desarrollan el concepto del "triple bottom line" acompañado de sus ejes conceptuales "people, planet, profit" (Elkington, 1999).

\section{Desarrollo sostenible y sector empresarial}

En 1995, aparece de nuevo el Consejo de Negocios para el Desarrollo Sostenible, como resultado de identificar la necesidad de mantener el vínculo empresarial participando activamente en la definición de las políticas emergentes de gestión ambiental, esta vez bajo el nombre de Consejo Mundial de Industria y Ambiente (WICE, su sigla en inglés) radicado en París e institucionalizado como adscrito a la Cámara de Comercio Internacional; que estableció luego su sede principal en Ginebra con su actual nombre: Consejo Mundial de Negocios para el Desarrollo Sostenible (WBCSD, por su sigla en inglés) (1997).

Este mismo año la Shell sin proponérselo se convirtió en el símbolo de la gestión en aspectos ambientales y sociales del triple bottom line, por causa de las controversias generadas por su actuación en Nigeria y en el Brend Spar, actuaciones que hacen referencia a una masacre en la que se presume la participación de empleados de la Shell; en un acto de desalojo de una comunidad local próxima a una línea de conducción de crudo, y el caso Brend Spar, uno de los más discutidos, relacionado con plataformas petroleras en el mar del Norte y sus efectos nocivos sobre el medio ambiente debido a mala disposición de desechos y filtraciones de crudo. En la actualidad esta plataforma ha sido desmantelada.

La empresa SustanAbility trabaja con otras compañías destacadas mundialmente para dilucidar las implicaciones futuras de estos eventos, pero declina la invitación extendida por Shell para trabajar directamente con ellos.

En 1996 la International Standard Organization (ONG creada bajo el auspicio del Plan Marshall para la reconstrucción del aparato productivo de los países devastados por la Segunda Guerra Mundial), emitió el conjunto de normas ISO 14000 para la creación e implementación de sistemas de gestión ambiental de adopción voluntaria, el cual tomó como referencia la estructura de su ya exitosa y ampliamente difundida norma para el establecimiento de sistemas de aseguramiento de calidad ISO 9000. Esta nueva norma se guió además por la BS7750 simplificando sus contenidos, la forma de los informes y haciéndola voluntaria lo que favoreció su adopción por empresas alrededor del mundo (Robinson y Roberts, 1998).

Este mismo año, el Departamento de Energía de Estados Unidos publicó la resolución DOE.P 450-4 con la que reglamentaba la constitución, diseño e implementación de los sistemas integrados de gestión (IMS, por su sigla en inglés), que luego se normatizarían y evolucionarían en la forma del Es\&H. Este es un modelo de sistema integrado de gestión concentrado en medio ambiente, seguridad y salud ocupacional, orientado a la aplicación de medidas de prevención y seguridad en puestos y ambientes de trabajo.

El año siguiente, en 1997 se celebró la Cumbre Río+5 en Nueva York. Se establecie- 
ron cuotas y medidas para detener el calentamiento global. El presidente Bill Clinton firmó los acuerdos, pero estos no fueron ratificados por el Congreso de los Estados Unidos. En esta misma reunión se hizo un balance de los pactos logrados a partir de la firma de la Cumbre de Río y se descubrió que, a pesar de la expansión en la adopción de medidas de gestión ambiental en todos los países, los resultados sobre la contaminación global no son significativos.

Se establecieron acuerdos para endurecer las políticas regionales y locales referentes a la contaminación y su control desde las fuentes de origen (industria). Se establecieron también los lineamientos generales para hacer del control de emisiones un negocio susceptible de transacciones en el sistema financiero internacional y se gestaron las bases del ecobanking como medida impulsora de estos tratados.

Durante ese mismo año, la empresa SustainAbility publicó el libro Cannibals with Forks, que se convirtió rápidamente en texto de referencia para la difusión y puesta en marcha de la agenda del triple bottom line en empresas alrededor del mundo. En forma simultánea, esta empresa consultora inició actividades con Shell, como consejera en sus nuevos reportes empresariales y en la definición de su nueva estrategia relacionada con la implementación del triple bottom line. Al mismo tiempo, Ceres hacía el lanzamiento del Global Reporting Initiative (GRI), como un estándar internacional para el reporte corporativo del desempeño económico, ambiental y social de las empresas.

En 1998, UNEP y Shell publicaron el libro The Social Reporting Report con la participación de SustainAbility. Asimismo esta empresa de consultoría fue invitada por las directivas de la Ford Motor Company para trabajar con ellos, y cancelaron relaciones con Monsanto debido a su negativa a atender las tensiones sociales en Europa generadas por su paquete tecnológico GM (Genéticamente modificado -Transgénicos).

En 1999 aparecieron nuevas publicaciones de SustainAbility, entre ellas The New Food Guide, con la cual oficializaron su interés corporativo por atender sectores de alimentación, agricultura y salud, así como las prácticas de trazabilidad en las cadenas de suministros. La trazabilidad se entiende como procedimientos preestablecidos y autosuficientes que permiten conocer, la ubicación y la trayectoria de un producto a lo largo de la cadena de suministros, mediante determinadas estrategias. Otras de sus publicaciones son los reportes sectoriales para la industria del petróleo y automotriz, y de ciencias de la vida. Estas publicaciones sentaron las bases para The Global Reporters, en 2000 que compara todos los sistemas de reporte de actividad empresarial relacionados con desarrollo sostenible.

Ese mismo año, la organización The Natural Step recibió el premio Millenium Mikhail Gorbachov, por sus aportes al logro del desarrollo sostenible en el sector productivo, el cual fue reafirmado con el premio The Blue Planet en 2000, considerado como el premio Nobel de la gestión ambiental.

En ese mismo periodo, gracias a la vinculación de la UNEP, el GRI adquirió una dimensión global con motivo de la conformación de una red de expertos en desarrollo sostenible alrededor del mundo, que incluye representantes de otros órga- 
nos como WBCSD, OeCD, DTIE (División de Tecnología, Industria y Economía de UNEP), y directores de empresas y oficinas gremiales internacionales de contabilidad y responsabilidad social corporativa.

En el año 2001 fue publicada la norma actualizada para países de la Comunidad Europea de Naciones -EMAS II-, que establece su adopción obligatoria a todo el sector productivo de la comunidad, en un formato compatible con los requerimientos y especificaciones de la norma ISO 14000, con el ánimo de facilitar la homologación de diferentes sistemas de gestión ambiental y su integración con otros sistemas de gestión existentes en el ámbito industrial (ISO 9000/2000, BASC, OHSAS 18000, entre otras).

Ese mismo año, SustainAbility publicó The Crysalis Economy (Elkington, 2001), concentrándose en la necesidad de crear y recuperar valores corporativos. Otra de sus publicaciones, también en 2001 Buried Treasure, que se constituyó en uno de los primeros reportes de casos de responsabilidad corporativa y desarrollo sostenible. Adicionalmente, con la participación del foro internacional de líderes de negocios (IBLF, por su sigla en inglés), esta empresa de consultoría ambiental publicó The Power to Change, que introduce los principios "Leader" para el fomento del elevado desempeño empresarial en desarrollo sostenible.

En 2002 se celebró la segunda Cumbre Mundial del Medio Ambiente y Desarrollo de la ONU, en Johannesburgo, sin la presencia de Estados Unidos. El tema central fue la reivindicación del desarrollo social y la problemática de los derechos de propiedad intelectual alrededor de recursos naturales, pero no logró avances significativos. Se hizo evidente la importancia de los sistemas de gestión ambiental en las empresas, como gestores del saneamiento ambiental y la necesidad de su integración con los sistemas de gestión empresarial asociados al desarrollo social.

También se realizó el Foro Económico Mundial en Nueva York, con la participación de SustainAbility, empresa que publicó ese año junto con la UNEP Good Mews and $\mathrm{Bad}$, que es una primera aproximación a la relación de los medios de comunicación con el desarrollo sostenible. Y también se publicó Trust Us, un segundo estudio sobre los sistemas de reporte corporativo y desarrollo sostenible.

Además se publicó Developing Value, un estudio de caso desarrollado con la ayuda de la Corporación Financiera Internacional (IFC, por su sigla en inglés) y el Instituto Ethos, que concentra sus estudios en economías emergentes.

Este mismo año, Ceres presentó su proyecto de gobierno corporativo para la sostenibilidad, con el objetivo de incrementar el compromiso de las empresas y entidades fiduciarias con relación a temas urgentes de carácter ambiental como el cambio climático. En forma paralela, el GRI adquirió su actual categoría de ONG de alcance global e independiente con oficinas centrales en Holanda y más de setecientas empresas adscritas

\section{Gestión ambiental y bienestar social}

En 2003 se realizó el primer Foro Social Mundial en Brasil, donde SuatainAbility participó con el lanzamiento de su libro The 21st Century NGO: In the Market for Change, el cual fue realizado conjuntamente con la oficina Global Compact de la ONU. De igual modo, se hizo evidente un aumento del inte- 
Este inventario de hechos pone de manifiesto que la gestión ambiental está evolucionando hacia sistemas de gestión que involucran variables relacionadas con el bienestar social. rés sobre los conceptos del Triple Bottom Line en la región Asia-Pacífico, donde Toyota se convirtió en la primera empresa en adoptar dichas prácticas y reportes en esta región.

En 2004 la norma ISO 14001 sufrió su primera modificación consistente en un cambio en la estructura del documento, con el ánimo de facilitar su integración a otros sistemas de gestión como el ISO 9000 y el OHSAS 18000 Fundación Confemetal (2005). De esta forma se inició un nuevo ciclo en la gestión, la cual empezó a integrar las áreas relacionadas con el desarrollo sostenible dentro de la empresa como lo son la gestión social, la ambiental y la económica, a pesar de contarse ya con experiencias previas como Triple Bottom Line, The Natural Step y Environment, Safety \& Health (ES\&H), ya comentadas.

Este mismo año, SustainAbility, junto con la fundación Mistra, publicaron Values for Money, que analiza el futuro de los fondos Socially Responsable Investing (SRI). Asimismo, un texto más de su serie de reportes de sostenibilidad corporativa Risk and Opportunity; así como el texto Gearing Up: From Corporate Responsability to Good Governance and Scalable Solutions, en un trabajo conjunto con la agencia Global Compact de la ONU.

En 2005 se realizó el Foro Económico Mundial en Davos (Suiza), y el Foro Social Mundial, en Porto Alegre (Brasil). Estos dos eventos contaron con la participación de SustainAbility.

Ese mismo año Karl-Henrik Robert, fundador de The Natural Step recibió la Primer Medalla Laureada a la Responsabilidad Social, otorgada por el Centro Global para el Liderazgo y la Ética en los Negocios.

Con estos eventos se formalizó la preocupación internacional por asuntos socia- les vinculados al desarrollo económico, colocando en un segundo plano los temas medioambientales que no dejan de ser preocupantes.

Este inventario de hechos pone de manifiesto que la gestión ambiental está evolucionando hacia sistemas de gestión que involucran variables relacionadas con el bienestar social.

En las últimas décadas del siglo XX se presentaron también algunas iniciativas que involucraron a los centros académicos y que dieron como resultado algunas iniciativas de gestión particulares para centros académicos siendo los más relevantes:

- Asociación Internacional de Uni-versidades (IAU, por su sigla en inglés), fundada en 1950 (1993).

- Universidad de Bolonia University of Bologna - European University Association (1987).

- Cooperation Programme in Europe for Research on Nature and Industry through Coordinated University Studies (Copernicus Campus, 2007).

- Declaración de Tallories (The Talloires Declaration, 2006).

- Universidades por un Futuro Sostenible (UlSF, 2008).

- Fundación Oikos (Oikos, 1990; Unced, 1992).

- Organización de Universidades por el Desarrollo Sostenible y el Medio Ambiente (OIUDSMA 1995).

- Universidad en Tokio, (UNU-IAS, 1994)

- Asociación Ambiental de Universidades y Colegios (EAuC, 1996).

- Grupo People and Planet (P\&P, 2004)

- Organización de las Naciones Unidas para la Educación, la Ciencia y la Cultura Unesco (ESD, 1995). 
Para continuar con el tema, en el próximo artículo se analizará la problemática ambiental y los centros de desarrollo académico. El
Politécnico Grancolombiano ha venido trabajando el tema desde el año 2005 (Camacho Delgado, 2006, 2007, 2008).

\section{Bibliografía}

1. Bisetti, A. (1988). Bernardino Ramazzini and occupational lung medicine. Annals of the New York Academy of Sciences, 534, 1029-37.

2. Bruntland, G. (1987). Our Common Future, Report of the World Commission on Environment and Development. World Commission on Environment and Development, 1987. Published as Annex to General Assembly document A/42/427. Development and International Cooperation: Environment (2 de agosto).

3. Camacho Delgado, Clemencia. (2008). Desarrollo sostenible: un tema de responsabilidad social universitaria, Compromiso del Politécnico Grancolombiano. En Responsabilidad Social de las Universidades. Tomo II. Buenos Aires: Red Latinoamericana de Cooperación Universitaria.

4. Camacho Delgado, Clemencia. (2007). La crisis del medio ambiente. En Revista Panorama (2) (agosto).

5. Camacho Delgado, Clemencia (2006). Propuesta de implementación de un sistema de gestión ambiental para Campus Universitario. En Poliantea (3) (diciembre).

6. Carson, R. (1962). Silent Spring. Houghton Mifflin, Mariner Books.

7. Clarence, J. (1956). Changing Ideas of the Habitable World. En Thomas, W. ed. Man's Role in Changing the Face of the Earth. Chicago University Press.

8. Commoner, B. (1971). The Closing Circle: Nature, Man, and Technology. Nueva York: Knopf.

9. Copernicus Campus. (2007). Copernicus-Guidelines for Sustainable Development in the European Higher Education Area [On line] Copernicus. [Disponible en:] www.dcsf.gov.uk/londonbologna/uploads/documents/COPERNICUSGuidelines. pdf (Consultado el 1 de noviembre de 2006).

10. Dasman, R. (1965). The destruction of California. Nueva York: Collier Books.

11. Dobson, Andrew. 1999. Pensamiento verde una antología. Uruguay. Editorial Trotta.

12. EAUC. (1996). The Enviromental Association of Universities and Collleges. Who we are. [Online]. EAUC. [Disponible en:] http://www.eauc.org.uk/about_us (Consultado 18 de octubre de 2006).

13. Elkington, J., Burke, T. (1987). The Green Capitalists: Industry's Search for Environmental Excellence. London: V. Gollancz.

14. Elkington, J. (1999).Cannibals with Forks: The Triple Bottom Line of 21st Century Business. London: V. Gollancz.

15. Elkington, J. (2001). He Chrysalis Economy: How Citizen CEOs and Corporations Can Fuse Values and Value Creation. London: V. Gollancz. 
16. ESD. (1995). Education for Sustainable Development. [Online]. Unesco. [Disponible en:] http://portal.unesco.org/education/en/ev.php-URL_

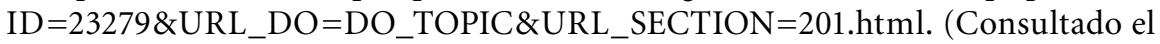
1 de noviembre de 2006).

17. Fundación Confemetal. (2005). Cómo implantar un sistema de gestión ambiental según Norma ISO 14001:2004. Taxus. Gestión Ambiental, Ecología y Calidad. Ed.

18. Griffiths, H. (2005). Human and Environmental Rights: The Need for Corporate Accountability. En Dodds, F. y Pippard, T. (eds.). Human \& Environment Security. An Agenda for Change. Londres: Earthscan.

19. Hook, G. (1995). Ramazzini; Father of Environmental Health. Environmental Health Perspectives. Tomo 103. (11) (noviembre).

20. Hutchinson, A. y Hutchinson, F. (1997). Environmental Business ManagementSustainable Development in the New Millennium. Londres: McGraw-Hill.

21. IAU. (1993). International Asociacion od Universities. Sustainable Development. Introduction. [Online] IAU. [Disponible en:] http://www.unesco.org/iau/sd/ index.html. (Consultado: 1 de noviembre de 2006).

22. Kapp, W. (1950). The Social Costs of Private Enterprise, Cambridge, Mass.: Harvard University Press.

23. Kapp, W. (1950a). The Social cost of Private Enterprice. Cambrige Massachusetts. Harvard University Press. En Lamprecht, J. Ed. ISO 14000, Issue \& Impementation Guideness for Responsible Environmental Management. (1997). Aenor.

24. Lamprecht, J. (1997). ISO 14000, Issue \& Impementation Guideness for Responsible Environmental Management. Aenor.

25. Makover, J., Elkington, J., y Hailes, J. (1993). The Green Consumer: Revised Edition. Londres: A Tilden Press Book.

26. McCormick, J. (2002), Environmental Policy in Britain. En Desai, U., (ed.), Environmental Politics and Policy in Industrialized Countries. Londres: Massachusetts Institute of Technology.

27. Marine, G. (1969). America the Raped: The engineering mentality of the devastation of a continent. Nueva York: Avon Books.

28. Massingham, H. (1942). The English Countryman: A study of The English Tradition. En Hutchinson, A y Hutchinson, F. Ed. Environmental Business Management. (1997). Londres: Mcgraw Hill.

29. Mayr, E. (1981). The Growth of Biological Thought. Cambridge: Harvard.

30. Mumford, L (1961). La condición del hombre. Buenos Aires: Compañía General Fabril Editora.

31. Muir, J. (1962). The Yosemite. Nueva York: Doubleday.

32. Muñoz, A. (2004). Universidad Sostenible. Avances de tecnología y producción (2). Bogotá: Universidad Externado de Colombia.

33. Naredo, J., Parra, F. (1993) Hacia una ciencia de los recursos naturales. Madrid: Siglo XXI España Editores (primera edición).

34. Oikos. (1990). Oikos Foundation. [Online]. Oikos International. [Disponible en:] http://www.oikos-international.org/about-oikos/organisation/oikos-foundation. html (Consultado: 1 de noviembre de 2006). 
35. OUIDSMA. (1995) Organización Inernacional de Universidades pro el Desarrollo Sostenible y el Medio Ambiente. Declaracion de Compromisos Universitarios para el Desarrollo Sosteible. [Online]. OuIDSMA. [Disponible en]: http://www. oiudsma.org/page.php?pageid=compromisos (Consultado: 10 de junio de 2003).

36. P\&P. (2004). People \& Planet - Going Green Report - Executive Summary. [Online]. P\&P. [Disponible en:] http://peopleandplanet.org/gogreen/report.php (Consultado: 27 de octubre de 2006).

37. Pearce, D., Markandya, A., Barbier, E. (1989). Blueprint for a Green Economy. (Blueprint 1). Londres: Earthscan.

38. Ponting, C. (1991). A Green History or the World. Nueva York: Penguin Books.

39. Reich, C. (1970). The Greening of America. Nueva York: Bantam Books.

40. Robinson, G. \& Roberts, H. (1998). ISO 14000 EMS Implementation Handbook, 1998.

41. The Tallories Declaration. (2006?). The Talloires Declaration: University Presidents for a Sustainable Future [Online] [Disponible en:] http://www.iisd.org/educate/ declarat/talloire.htm. (Consultado: 4 de septiembre de 2006).

42. Thomas, W. Jr. (1956). Man's Role in Changing the Face of the Earth. Chicago, Chicago University Press. (Nota: recopilación de las memorias del evento realizado en 1955; más de 1.200 páginas).

43. Tippett, J. (2005). The Value of Combining a Systems View of Sustainability with a Participatory Protocol for Ecologically Informed Design in River Basins. Journal of Environmental Modeling and Software, 20 (2), 119-139.

44. UlSF. (2008). About ULSF. [Online]. ULSF. [Disponible en:] http://www.ulsf.org/ about.html. (Consultado: 1 de noviembre de 2008).

45. Unced. (1992). United Nations Conference on Environment and Development. [Online]. Unced. [Disponible en:] http://www.ciesin.columbia.edu/TG/PI/ TREATY/unced.html. (Consultado: 1 de noviembre de 2006).

46. University of Bologna - European University Association. (1987). Magna Charta Universitarium. [Online]. Observatory Magna Charta Universitarium. [Disponible en:] http://www.magna-charta.org/magna.html. (Consultado: 4 de septiembre de 2006).

47. UnU-IAS (1994). UnU/Zero Emitions Research Initiative. [Online]. UNU. Disponible en:] http://www.ias.unu.edu/sub_page.aspx?catID $=5 \&$ ddlID $=468$ (Consultado: 1 de noviembre de 2006).

48. Vogel, D. (1990). Environmental Policy in Europe and Japan. En Vig, N. y Kraft, M. Ed. Environmental Policies in 1990's. Washington D.C.: CQ Press.

49. WBCSD. (1997). Hystory of the WBCSD. [Online] WBCSD. [Disponible en:] http:// www.wbcsd.org/templates/TemplateWBCSD2/layout.asp?type $=\mathrm{p} \&$ MenuId $=\mathrm{ND}$ Ex\&doOpen=1\&ClickMenu=LeftMenu. (Consultado: 19 de julio de 2006).

50. Wolman, A. (1956). Disposal of Man's Waste. En Thomas, W. Jr. ed. Man's Role in Changing The Face of the Earth. Chicago: Chicago University Press. 